Z. Klin. Chem. Klin. Biochem.

11. Jg. 1973, S. $297-300$

\title{
Lipidzusammensetzung gesunder und pathologischer Hautbezirke bei Psoriasis vulgaris
}

\author{
Von J. Jacob und G. Grimmer \\ Biochemisches Institut für Umweltcarcinogene, Abrensburg/Holstein
}

(Eingegangen am 8. Dezember 1972/16. März 1973)

\begin{abstract}
Die Oberflächenlipide gesunder und kranker Hautpartien (Schuppen) von Patienten mit Psoriasis vulgaris wurden untersucht und mit denen gesunder Probanden verglichen. Die Struktur der Fettsäuren mit einer und zwei Doppelbindungen führt zu dem Schluß, daß die Fettsäuren der Hautoberfläche dem Talg entstammen, während die aus Schuppen extrahierten Fettsäuren den Lipidanteilen von Zellmembranen entstammen.
\end{abstract}

\section{The lipid composition of bealtby and patbological skin areas in Psoriasis vulgaris}

Lipids from the surface of healthy areas and affected areas (scales) of patients suffering from psoriasis vulgaris were investigated and compared with those of healthy people.

The analysis of the monoenoic and dienoic fatty acids indicates that the skin surface fatty acids originate from the sebum, whereas fatty acids extracted from the scales originate from the lipid parts of the cell membranes.

Wir haben in einer vorangehenden Arbeit über die Unterschiede in der Isomerenzusammensetzung von Monoen- und Dienfettsäuren aus gesunder Haut. und Psoriasisschuppen berichtet (1). Es erschien uns aufgrund der dort erhobenen Befunde notwendig $\mathrm{zu}$ überprüfen, ob sich diese Unterschiede auch an gesunden und erkrankten Bezirken eines und desselben Patienten bestätigen lassen.

Aufgrund der Tatsache, daß es sich bei dem Oberflächenfett (Sebum), das von den Talgdrüsen produziert wird, und bei den Lipiden der Psoriasisschuppen, von denen anzunehmen ist, daß sie vornehmlich Gerüstlipide darstellen, um zwei verschiedene Fettarten handelt, war zu erwarten, daß sich dieses auch in der Feinstruktur der Fettsäuren dokumentieren würde. Um Veränderungen dieser Lipide gegenüber denen gesunder Probanden festzustellen, wurden gleichzeitig von diesen Sebumproben gesammelt und untersucht, obschon hierüber zahlreiche Veröffentlichungen bereits vorliegen. Über eine Analyse der Lipide aus dem stratum corneum, das mit Psoriasisschuppen am ehesten zu vergleichen ist, wurde kürzlich von ANSARI und Nicolardes (2) berichtet.

\section{Material und Methoden}

Für den Vergleich gesunder und von Psoriasis befallener Hautpartien stand uns eine an Psoriasis vulgaris erkrankte Patientin zur Verfügung, die 8 Wochen vor der Probennahme von jeder Therapie zurückgestellt wurde, um eine Beeinflussung des Lipidstoffwechsels der Haut weitgehend auszuschließen. Von erkrankten Bezirken wurden Schuppen gesammelt $(1,2264 \mathrm{~g})$. Fettmuster gesunder Stirnpartien (Stirn wurde nur mit Wasser gewaschen; es wurden keine Cremes benutzt) wurden durch 8stündiges Auflegen von Zigarettenpapier gewonnen, aus dem die Lipide anschließend extrahiert wurden. Gleichzeitig wurde das eingesetzte Papier untersucht, um Verunreinigungen aus dieser Quelle zu eliminieren. Zum Vergleich wurde ferner von vier gesunden
Probanden durch Abreiben der Stirn mit Aceton Stirnfett gesammelt.

\section{Extraktion}

Die Extraktion der Rohlipide erfolgte mit $60 \mathrm{ml} \mathrm{CHCl} / \mathrm{CH}_{3} \mathrm{OH}$ $(40 \mathrm{ml}+20 \mathrm{ml})$. Nach Zugabe von Wasser $(20 \mathrm{ml})$ wurde die organische Phase im Vakuum bei $30^{\circ} \mathrm{C}$ Badtemperatur eingedampft (Ergebnisse in Tab. 1).

\section{Dïnnscbichtcbromatograpbie}

Die Dünnschichtchromatographie der Rohlipide wurde an Kieselgel-Fertigplatten (E. Merck) im System $\mathrm{CHCl}_{3}$ (System I) bzw. $\mathrm{CHCl}_{3} / \mathrm{CCl}_{4} 10 \mathrm{ml}+10 \mathrm{ml}$ (System II) durchgeführt und zeigt für die Rohlipide gesunder und erkrankter Hautbezirke deutliche Unterschiede. In den Schuppenlipiden dominieren Koblenwasserstoffe $\left(R_{F(I I)}=0,95\right)$, Cholesterin $\left(R_{F(I)}=0,23\right)$ und freie Alkohole $\left(R_{F(I)}=0,3\right)$, während die Lipide aus der gesunden Haut hauptsächlich aus Squalen $\left(R_{F(I I)}=0,89\right)$, Wachsen und Triglyceriden $\left(R_{\mathbf{F}(1)}=0,8\right.$ und 0,45$)$ bestehen. Dagegen weist das gleichzeitig untersuchte Papier nur Spuren polarer Bestandteile auf. Die Detektion erfolgte durch Ansprühen mit Chromschwefelsäure und anschließendes Erhitzen auf $150^{\circ} \mathrm{C}$.

\section{Säulencbromatographie}

Die Rohlipide wurden durch eine Säulenchromatographie an Kieselgel (E. Merck, $\mathrm{CHCl}_{3}$-gewaschen, Korngröße 0,16 mm, 50 facher Uberschuß) mit $\mathrm{CHCl}_{3}$ in unpolare und polare Lipide getrennt. Im $\mathrm{CHCl}_{3}$-Eluat befinden sich hierbei die Kohlenwasserstoffe, Cholesterinester, Wachse, Triglyceride, Alkohole, das Cholesterin und die freien Fettsäuren, während die Phosphatide mit $\mathrm{CHCl}_{3} / \mathrm{CH}_{3} \mathrm{OH}(10 \mathrm{ml}+10 \mathrm{ml})$ eluiert werden.

Die unpolaren Lipide wurden in der gleichen Weise $(10 \mathrm{~g}$ Kieselgel Woelm, $8 \%$ Wassergehalt) weiter fraktioniert, wobei zur Elution ein $\mathrm{CCl}_{4} / \mathrm{CHCl}_{3}$-Gradient in der Rcihenfolge $\mathrm{CCl}_{4}, \mathrm{CCl}_{4}$ mit 5, 10, $20,40,60,80 \% \mathrm{CHCl}_{3}$ benutzt wurde. Die Elutionsmittelmenge betrug jeweils $40 \mathrm{ml}$, während die Eluate in 5 ml-Fraktionen gesammelt und ihre Zusammensetzung dünnschichtchromatographisch untersucht wurden.

\section{Gaschromatograplie}

Alle Fraktionen wurden als solche gaschromatographisch untersucht, zusammengesetzte Fraktionen (Wachse, Cholesterinester, 
Triglyceride) wurden, wie früher beschrieben (3), mit 5proz. methanolischer $\mathrm{HCl}$ umgeestert und die resultierenden Methylester von den Alkoholen bzw. vom Cholesterin säulenchromatographisch (4) abgetrennt. Die Bedingungen der Gaschromatographie haben wir früher beschrieben (3).

\section{Identifikation der Komponenten}

Die Identifikation dex in den Tabellen angegebenen Fettsäuren (bzw. deren Methylester) erfolgte durch Vergleich dex Retentionszeiten mit denen authentischer Fettsäuremethylester, soweit sie verfügbar waren, auf verschieden polaren Säulen (Polydimethylsiloxan, Diäthylenglykolsuccinat-polyester). Zum Vetgleich standen uns folgende Fettsäuremethylester zur Verfügung: $\mathrm{C}_{10: 0}, \mathrm{C}_{11: 0}, \mathrm{C}_{12: 0}, \mathrm{C}_{13: 0}, \mathrm{C}_{14: 0}, \mathrm{C}_{15: 0}, \mathrm{C}_{16: 0}, \mathrm{C}_{17: 0}, \mathrm{C}_{18: 0}$,

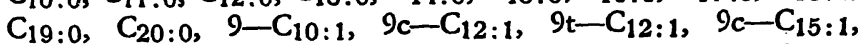
$9 c-C_{16: 1}, 9 c-C_{17: 1}, 9 c-C_{18: 1}, 6 c-C_{15: 1}, 6 c-C_{16: 1}$, $7 c-C_{13: 1}, 7 c-C_{15: 1}, 7 c-C_{16: 1}, 10 c-C_{15: 1}, 6 c, 9 c-C_{18: 2}$ $6 c, 12 c-C_{18: 2}, 8 c, 11 c-C_{18: 2}, 9 c, 12 c-C_{18: 2}$, sowie eine große Anzahl isomerer Monoenfettsäuren, die im Hautfett nicht nachgewiesen werden konnten. Daneben standen uns zahlreiche methylsubstituierte Fettsäuremethylester zur Verfügung. Alle nicht verfügbaren Fettsäuremethylester wurden nach der Methode der äquivalenten Kettenlänge gaschromatographisch zugeordnet.

Die Monoenfettsäuren wurden nach Hydrierung mit $\mathrm{PtO}_{2} / \mathrm{H}_{2}$ in Methanol zusätzlich massenspektrometrisch identifiziert. Ihre Struktur ergibt sich außerdem aus der oxidativen Spaltung.

Ebenfalls massenspektrometrisch wurden die methylsubstituierten Fettsäuren identifiziert, über deren Zusammensetzung später gesondert berichtet werden soll.

\section{Massenspektrometrie}

Die Gaschromatographie/Massenspektrometrie-Kombination wurde an dem Massenspektrometer GNOM (Vatian MAT 111) mit einer 9m-Glassäule (Methylsilikon OV 101, 5\% an GasChrom Q, $100-120$ mesh, Durchm. 2,8 mm) bei $80 \mathrm{eV}$ Anregungsspannung und $5 \cdot 10^{-6}$ Torr durchgeführt. Säulen-, Trenner- und Injektionstemperatur betrugen $200^{\circ} \mathrm{C}$. Die Strömungsgeschwindigkeit entsprach bei einem Vordruck von 3 atü etwa $20 \mathrm{ml}$ Helium/min. Die Detektion erfolgte durch Messung des Totalionenstromes (Beschleunigungsspannung $820 \mathrm{~V}$ ).

\section{Trennung der Fettsäuremetbylester nach dem Grad der Sättigung}

Die Trennung dex Triglycerid-Fettsäuren aus dem Hautfett und den Psoriasis-Schuppen nach dem Grad der Sättigung erfolgte nach dem von JANTZZEN et al. $(5,6)$ beschriebenen Verfahren über die $\mathrm{Hg}[\mathrm{I}]$-acetat-Addukte. Details der Aufarbeitung haben wir früher beschrieben (7).

\section{Präparative Gascbromatograpbie}

Die Bedingungen der präparativen Gaschromatographie haben wir bereits mitgeteilt (3); sie lieferte die positionsisomeren Hexadecen-, Octadecen- und Octadecadien-säuremethylester in 99,9proz. Reinheit.

\section{Oxidative Spaltung}

Die oxidative Spaltung wurde von uns an anderer Stelle ausführlich beschrieben $(8,9)$.

\section{Ergebnisse und Diskussion}

Die Ergebnisse der Aufarbeitung des Materials und der prozentualen Verteilung der Bestandteile auf die einzelnen Lipidklassen sind in Tabelle 1 wiedergegeben.

Das gleichzeitig untersuchte Zigarettenpapier enthielt $0,2 \%$ extrahierbare Lipide, die ausschließlich aus

Tab. 1

Lipidzusammensetzung der Psoriasisschuppen und des Hautfetts gesunder Hautbezirke

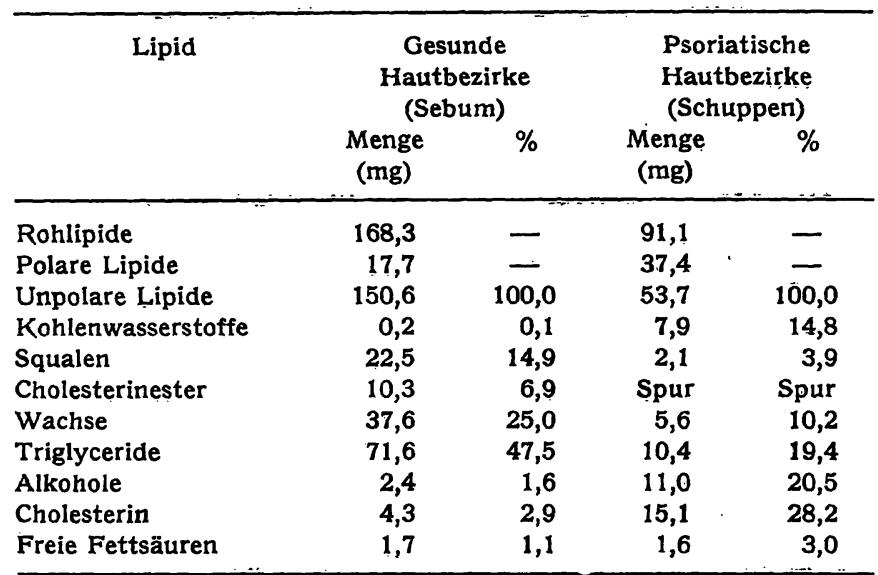

Tab. 2

Fettsäurezusammensetzung einzelner Lipide gesunder und psoriatischer Hautbezirke (in \%)

\begin{tabular}{|c|c|c|c|c|c|c|c|c|}
\hline \multirow[t]{2}{*}{ Fettsäure } & \multicolumn{2}{|c|}{ Cholesterinester } & \multicolumn{2}{|c|}{ Wachse } & \multicolumn{2}{|c|}{ Triglyceride } & \multicolumn{2}{|c|}{ Freie Fettsäuren } \\
\hline & (Sebum) & (Schuppen) & (Sebum) & (Schuppen) & (Sebum) & (Schuppen) & (Sebum) & (Schuppen) \\
\hline $10: 0$ & 0,8 & Spur & Spur & 一 & 0,4 & 0,3 & 0,5 & Spur \\
\hline $11: 0$ & Spur & Spur & Spur & - & Spur & 0,1 & 0,7 & Spur \\
\hline $12: 0$ & 0,7 & 2,0 & Spur & Spur & 2,4 & 0,3 & 2,7 & 0,3 \\
\hline $12: 1$ & 1,5 & 0,2 & 12,2 & 0,1 & 0,6 & 0,1 & 1,0 & Spur \\
\hline $13: 0$ & Spur & Spur & Spur & - & 0,9 & 0,3 & 1,1 & 0,3 \\
\hline unbek. & 1,0 & 0,3 & - & - & 1,8 & 0,3 & 3,2 & 0,1 \\
\hline $14: 0$ & 5,4 & 6,0 & 0,2 & 2,0 & 14,3 & 3,2 & 14,6 & 2,3 \\
\hline $14: 1$ & 11,3 & 1,2 & 17,4 & 0,5 & 6,5 & 0,5 & 0,9 & 0,4 \\
\hline $15: 0$ & 4,6 & 3,0 & 4,4 & 1,0 & 7,8 & 3,6 & 2,1 & 1,3 \\
\hline $15: 1$ & 1,8 & 0,3 & 4,5 & 0,1 & 3,7 & 0,3 & 2,5 & 0,4 \\
\hline $16: 0$ & 11,7 & 23,0 & 6,2 & 24,6 & 25,7 & 15,4 & 34,5 & 15,0 \\
\hline $16: 1$ & 11,3 & 10,2 & 47,5 & 8,2 & 22,4 & $\cdot 10,2$ & 17,0 & 12,7 \\
\hline $17: 0$ & Spur & Spur & 3,4 & 1,0 & 1,5 & 0,9 & 0,8 & 0,8 \\
\hline $17: 1$ & Spur & 1,0 & 6,2 & 1,1 & 2,4 & 1,6 & 2,2 & 1,9 \\
\hline $18: 0$ & 0,6 & 11,0 & 0,7 & 12,0 & 1,4 & 6,8 & 10,3 & 3,2 \\
\hline $18: 1$ & 4,6 & 32,7 & $-3,7$ & 31,2 & 7,1 & 28,9 & 5,9 & 31,5 \\
\hline $18: 2$ & - & 7,7 & 1,9 & 18,2 & 0,9 & 25,5 & Spur & 28,8 \\
\hline $18: 3$ & Spur & 1,4 & - & Spur & - & 1,1 & - & 1,0 \\
\hline 19:0 & - & Spur & - & Spur & - & 0,6 & - & Spur \\
\hline $20: 0(?)$ & 14,4 & - & 1,1 & - & $=$ & - & - & - \\
\hline $20: 1$ & 2,4 & - & 0,6 & - & - & - & - & - \\
\hline $20: 4$ & 27,9 & - & - & - & - & - & - & - \\
\hline verzw. & Spur & - & Spur & - & Spur & - & Spur & - \\
\hline
\end{tabular}


polaren Anteilen bestanden. Ebenfalls abgenommene Stirnfettproben (54 Extraktionen) von 4 gesunden Probanden lieferten nach der Extraktion 784,9 mg Rohlipide, die zu $46 \%$ aus freien Fettsäuren bestanden. Die übrigen Lipide dieses Extraktes wurden nicht näher untersucht.

Während die gesunde Haut erhebliche Mengen Squalen $(14,9 \%)$ enthält, dessen Struktur durch Massenspektrometrie der originalen sowie der hydrierten Verbindung in der Gaschromatographie/MassenspektrometrieKopplung gesichert wurde, treten bei den Schuppenlipiden $n$-Paraffine $(14,8 \%)$ in den Vordergrund. Wir haben diese Befunde bereits in einer vorhergehenden Veröffentlichung (1) erhoben und darauf hingewiesen, $\mathrm{da} ß$ die Paraffine aus exogenen Quellen stammen könnten, obschon der hier untersuchte Patient über länger als 8 Wochen von jeglicher Therapie abgesetzt worden war.

Cholesterinester fehlen in den Schuppenlipiden praktisch vollständig, wohingegen Wachse, Triglyceride nur erniedrigt, freie Alkohole und freies Cholesterin erhöht und die freien Fettsäuren gegenüber dem Stirnfett (gesunde Haut) des gleichen Patienten unverändert sind.

Die Zusammensetzung der Fettsäuren aus den Cholesterinestern, Wachsen, Triglyceriden und den freien Fettsäuren ist in Tabelle 2, die der Wachs- und der freien Alkohole in Tabelle 3 wiedergegeben.

Bezüglich der Fettsäuren zeigen die Tabellen, daß in den pathologischen Bereichen Octadecen- und Octadecadiensäure stark erhöht sind. In den Cholesterinestern und Wachsen ist der Wert für die Palmitinsäure stark erhöht, in den Triglyceriden und freien Fettsäuren erniedrigt. Bei den freien Alkoholen und den
Wachsen zeigen sich Veränderungen in der gleichen Richtung: die geradzahligen langkettigen Alkohole sind in den Psoriasisschuppen deutlich erhöht.

Signifikante Unterschiede bezüglich der Doppelbindungslage finden sich auch bei den Hexadecen-, Octadecen- und Octadecadiensäure-Isomerengemischen der Triglyceride, die nach präparativer Gaschromatographie mit Hilfe der oxidativen Spaltung mit $\mathrm{MnO}_{4} / \mathrm{JO}_{4}$ analysiert wurden. Die Ergebnisse sind zusammen mit denen von NicolaIdes $(13,14,16)$ in Tabelle 4 wiedergegeben.

Die Kettenlängen der Mono- und Dienfettsäuren wurden ferner nach Hydrierung mit $\mathrm{PtO}_{2} / \mathrm{H}_{2}$ in $\mathrm{Me}$ thanol massenspektrometrisch bestimmt.

Normales Hautfett zeichnet sich durch einen hohen Gehalt (30\% und mehr, $46 \%$ in der vorliegenden Untersuchung) an freien Fettsäuren aus $(10,11,12)$,

Tab. 3

Zusammensetzung der freien und der Wachsalkohole der Lipide aus Psoriasisschuppen und Hautfett gesunder Bezirke (in \%)

\begin{tabular}{lcccc}
\hline \multicolumn{1}{c}{ Alkohole } & \multicolumn{2}{c}{ Freie Alkohole } & \multicolumn{2}{c}{ Wachsalkohole } \\
& (Sebum) & (Schuppen) & (Sebum) & (Schuppen) \\
\hline Decanol & 1,0 & 0,5 & 0,1 & 2,9 \\
Undecanol & 2,3 & 1,2 & 0,2 & 1,9 \\
Dodecanol & 1,6 & 0,2 & 0,2 & 0,8 \\
Unbekannt & 0,1 & Spur & 0,1 & 0,4 \\
Tridecanol & 8,4 & 1,1 & 19,7 & 0,9 \\
Tetradecanol & 4,4 & 0,4 & 17,8 & 4,0 \\
Pentadecanol & 45,6 & 1,6 & 51,0 & 3,8 \\
Hexadecanol & 6,5 & 15,4 & 10,1 & 42,0 \\
Unbekannt & 3,0 & 0,3 & 0,1 & Spur \\
Heptadecanol & 22,5 & 0,9 & 0,1 & 0,9 \\
Unbekannt & 0,1 & 0,5 & Spur & 0,4 \\
Octadecanol & 4,5 & 37,6 & 0,6 & 32,0 \\
Eicosanol & - & 40,3 & Spur & 10,0 \\
\hline
\end{tabular}

Tab. 4

Isomerenzusammensetzung (in Mol-\%) der Hexadecen-, Octadecen- und Octadecadiensăuren der Triglyceride aus Sebum und Schuppen des gleichen Psoriasiatikers

\begin{tabular}{|c|c|c|c|c|c|}
\hline Fettsäure & $\begin{array}{c}\text { Doppel- } \\
\text { bindungslage }\end{array}$ & $\begin{array}{l}\text { Schuppen } \\
\text { (Psoriasis) }\end{array}$ & $\begin{array}{c}\text { Sebum } \\
\text { (Psoriasis) }\end{array}$ & $\begin{array}{c}\text { Sebum } \\
\text { (1. c. 13) }\end{array}$ & $\begin{array}{l}\text { Sebum } \\
\text { (1. c. 14, 16) } \\
\end{array}$ \\
\hline \multirow[t]{8}{*}{$16: 1$} & 4 & Spur & 3,5 & - & - \\
\hline & 5 & - & Spur & - & 1 \\
\hline & 6 & 4,0 & 82,5 & 99 & 95 \\
\hline & 7 & 3,9 & 1,9 & - & 1 \\
\hline & 8 & 1,4 & 9,9 & - & 2 \\
\hline & 9 & 89,7 & 2,2 & - & 1 \\
\hline & 10 & 1,0 & Spur & - & - \\
\hline & 11 & Spur & Spur & - & -- \\
\hline \multirow[t]{8}{*}{$18: 1$} & 4 & - & 3,9 & - & - \\
\hline & 5 & - & 1,7 & - & 3 \\
\hline & 6 & - & 7,5 & 15 & 14 \\
\hline & 7 & 1,0 & 2,5 & - & 2 \\
\hline & 8 & 2,4 & 66,0 & 70 & 46 \\
\hline & 9 & 85,6 & 14,3 & 15 & 33 \\
\hline & 10 & 4,1 & 4,1 & - & 2 \\
\hline & 11 & 6,9 & Spur & - & - \\
\hline \multirow[t]{8}{*}{$18: 2$} & 4,7 & - & 6,8 & - & - \\
\hline & 5,8 & - & 17,3 & - & 55 \\
\hline & 6,9 & 0,2 & 13,9 & - & 5 \\
\hline & 7,10 & 1,3 & 23,0 & - & 2 \\
\hline & 8,11 & 4,2 & 29,6 & - & 4 \\
\hline & 9,12 & 89,8 & 17,3 & - & 26 \\
\hline & 10,13 & Spur & 2,1 & - & 8 \\
\hline & 11,14 & 4,5 & Spur & - & - \\
\hline
\end{tabular}


über deren Funktion nichts Sicheres bekannt ist. Die Fettsäuren stammen praktisch ausschließlich aus den Talgdrüsen und sind durch das Vorkommen von Monoenverbindungen mit den ungewöhnlichen Doppelbindungslagen in 6-, 8- und 10-Position sowie von Dienkomponenten mit 5,8-Doppelbindungslage charakterisiert $(2,10,13)$. Unsere Analyse des Stirnfettes gesunder Probanden, in dem 46\% freie Fettsäuren gefunden wurden, bestätigt die Untersuchungsergebnisse dieser Autoren. Als weiteres Charakteristikum kann das Vorkommen hoher Anteile von Squalen und Wachsen für das Hautfett herangezogen werden.

Die Hautlipide der gesunden Partien (Stirnfett) des hier untersuchten Patienten weisen im wesentlichen die Merkmale einer gesunden Haut auf: hoher Squalenund Wachsgehalt, sowie Vorkommen von hauptsächlich 6-, 8- und 10-Monoenfettsäuren. Auffällig ist dagegen der sehr geringe Anteil freier Fettsäuren am Gesamtlipidgehalt $(1,1 \%)$ und die Zusammensetzung der Linolsäuren, bei denen neben 5,8- vor allem 7,10und 8,11-Isomere beobachtet wurden. Sofern der niedrige Gehalt an freien Fettsäuren im vorliegenden Fall nicht überhaupt auf eine ungenügende Lipidversorgung der Psoriatiker-Haut hindeutet, ist zumindest die Abwehrlage derselben gegen Mikroorganismen verschlechtert.

Die Psoriasisschuppen zeigen schließlich eine Lipidzusammensetzung, die in guter Übereinstimmung mit den von ANSARI et al. (2) für das stratum corneum der menschlichen Epidermis mitgeteilten steht (etwa 70\% unverseifbare Anteile in beiden Fällen, ähnliche Verteilung der Fettsäuren, Vorherrschen von 9-Isomeren). Bemerkenswert ist auch hier zunächst der sehr niedrige Gehalt an freien Fettsäuren (3,0\%) und der schwierig zu deutende hohe Anteil an Kohlenwasserstoffen. Der reduzierte Squalengehalt und der damit offenbar im Zusammenhang stehende hohe Cholesteringehalt in den Schuppen gegenüber gesunder Haut zeigt, daß bei der Keratinisierung der Zellen die Squalensynthese gestoppt und alles synthetisierte Squalen $\mathrm{zu}$ Chol- esterin umgesetzt wird. Der hohe prozentuale Anteil an Phosphatiden sowie an Linolsäure (z. B. $25,5 \%$ der Triglyceridfettsäuren) an den Schuppenlipiden weist bereits darauf hin, daß es sich beim Sebum und bei den Schuppen um biogenetisch differente Lipide handelt. Die Schuppen weisen die typische Zusammensetzung von Zellmembranlipiden auf. Daneben stehen offenbar wenig frei verfügbare Fette den Zellen nach Abschluß der Keratinisierung zur Verfügung.

Auf eine unterschiedliche Zusammensetzung der Fettsäuren des Oberflächenfettes und der Schuppen von Psoriasispatienten ist von Rust (15) bereits hingewiesen worden. In der erwähnten Arbeit wurde aber die Feinstruktur der Fettsäuren nicht untersucht, die, wie die vorliegenden Untersuchungen zeigen, aber erst einen Einblick in die Lipidstruktur erlaubt. Sie beweist, daß es sich beim Sebum und den Lipiden der Schuppen, die ja nur durch unreguliertes Wachstum vermehrtes stratum corneum darstellen, um grundsätzlich andere Lipide handelt. Offenbar tritt bei der Differenzierung des Hautgewebes zur Talgdrüse - beide entstehen aus dem gleichen Epithelkeim - auch eine Differenzierung der Enzyme der Fettsäure- und Lipidbiosynthese ein.

Die Tatsache, daß in den Schuppen praktisch keine der für das Sebum charakteristischen Fettsäuren $\Delta^{6}-C_{16}$ und $\Delta^{8}-C_{18}$, sowie $\left.\Delta^{5,8}-C_{18: 2}\right)$ auftreten, spricht für eine schlechte Fettsäureversorgung dieser Hautbereiche. Dies wird auch durch die Tatsache unterstützt, daß nur geringe Mengen mit Fettsäuren veresterter Lipide beobachtet werden (niedriger Gehalt an Cholesterinestern, Wachsen und Triglyceriden, aber hoher Gehalt an freien Alkoholen und Cholesterin). Inwieweit diese schlechte Sebumversorgung ursächlich mit der Psoriasis verknüpft ist, läßt sich $z$. Z. nicht entscheiden, es ist jedoch anzunehmen, daß hierdurch die Abwehrlage der Haut des Psoriatikers geschwächt wird.

Der Deutschen Forschungsgemeinschaft danken wir für die Unterstützung dieser Arbeit.

\section{Literatur}

1. Grimmer, G., Jacob, J. \& Kimmig, J. (1971), diese Z. 9, 111-116. - 2. ANSARi, M. N. A., Nicolaides, N. \& Fu, H. C. (1970), Lipids 5, 838-845. - 3. JAСов, J. \& GRIMMER, G. (1970), Z. Naturforsch. B, 25, 54-56. - 4. JACOB, J. \& GLASER, A. (1970), Z. Naturforsch. B, 25, 1435-1437. - 5. JANTZEN, E. \& Andreas, H. (1959), Chem. Ber. 92, 1427-1437. - 6 . Jantzen, E., Andreas, H., Morgenstern, K. \& Roth, W. (1961), Fette, Seifen einschl. Anstrichm. 63, 685-688. - 7. JACOB, J. \& Grimmer, G. (1967), J. Lipid Res. 8, 308-311. 8. Grimmer, G. \& JACob, J. (1969), Z. Naturforsch. B, 24, 565-568. - 9. GrimMer, G. \& JACOB, J. (1969), Z. Natur- forsch. B, 24, 1004-1008. - 10. WeitKamp, A. W., Smiljanic, A. M. \& Rothman, S. (1947), J. Amer. Chem. Soc. 69, $1936-1939$. 11. HaAmtr, E., in: 'Major Lipid Constituents of Human Skin Surface' Mercatorin Kirjapaino, Helsinki 1961. - 12. Rothman, S. (1954), in: 'Physiology and Biochemistry of the Skin', University of Chicago press. - 13. NicolaIDES, N., KeLLUM, R. E. \& Wooliey III, P. V. (1964), Arch. Biochem. Biophys. 105, 634-639. 14. NicolaIdes, N. \& ANSARr, M. N. A. (1969), Lipids 4,79-81. 15. Rust, S., Harth, P. \& Herrmann, F. (1970), Arch. Klin. Exp. Derm. 238, 207-216. - 16. Nicolaides, N., Fư, H. C., Ansari, M. N. A. \& RiCE, G. R. (1972), Lipids 7, 506-517.

Prof. Dr. G. Grimmer

Biochemisches Institut für Umweltcarcinogene 207 Ahrensburg/Holst.

Sieker Landstr. 19 